\title{
A Research on Automatic Handwritten Devnagari Text Generation in Different Styles Using Recurrent Neural Network (Deep Learning) Especially for Marathi Script
}

\author{
Yogesh Kumar Sharma, Vajid Khan
}

\begin{abstract}
The point of handwritten numeral reputation (HNR) framework is to order input numeral all in all of $k$ classifications. There are standard HNR frameworks have 2 elements: handwritten numeral popularity. In spotlight exam step, data relevant as an example classifier. the example arrangement step names the numeral by means of and large of $k$ classifications exploitation the class models. in the course of the years, right savvy amount of labor has been allotted in the space of HNR. Fluctuated methods are arranged within the writing for characterization of composed numerals. those hold close Hough changes, visible diagram methods, head element research, and bolster vector machines, closest neighbor methods, neural figuring and fluffy essentially based totally methodologies
\end{abstract}

Keywords: Handwriting popularity, CNN-RNN community, records augmentation, photo pre-processing.

\section{INTRODUCTION}

The point of written with the aid of hand numeral acknowledgment (HNR) framework is to set up input numeral assembled of $\mathrm{k}$ training. There rectangular degree 2 everyday HNR frameworks have more than one components: spotlight investigation and example order. In highlight research step, records critical for instance classifier. the example order step names the numeral set up together of okay instructions abuse the magnificence models. all through the years, right terrific measure of labor has been appointed at interims the territory of HNR. Shifted approaches that square measure arranged at interims the writing for characterization of composed numerals. these draw close Hough modifications, bar graph methods that, head $1 / 2$ investigation, and bolster vector machines, closest neighbor systems, neural processing and fluffy primarily based simply techniques. An escalated assessment of acknowledgment execution for goliath composed facts thru a few types of options and classifiers is reportable. as contrasted and HNR frameworks of fluctuated non-Indian contents [e.g. Roman, Arabic, and Chinese] we discover that the prominence of composed numerals for Indian contents maintains on being a difficult assignment and there may be spurt for paintings to

Revised Version Manuscript Received on 16 September, 2019.

Dr.Yogesh Kumar Sharma, Professor, Research Coordinator JJTU Rajasthan, Deparment of Computer science and engineering, Head of Department, Research Coordinator Shri JJTU, Rajasthan, India.

(Email:dr.yogeshkumar@yahoo.in)

Mr.Vajid Khan, Research Scholar, Deparment of Computer science and engineering, Shri JJTU, Rajasthan, India

(Email:_kvajid12@gmail.com) be depleted this area. a brisk audit of work depleted acknowledgment of composed numerals written in Devanagari content material is given below .Devanagari content, to start with created to scribble down Sanskritic language, has slipped from the Brahmi content some time or any other round the eleventh century advertisement. it is custom-made to scribble down some Indo-Aryan dialects like Sanskritic language, Mundari, Nepali, Konkani, Hindi and Sanskritic language itself. Sanskritic language is associate diploma Indo-Aryan language spoken by using in regards to seventy a million people within the precept in the Indian province of Maharashtra and near states. Sanskritic language is likewise spoken in Israel and Mauritius. Sanskritic language is notion to be a descendent of Maharashtra, one in all the Prakrit dialects, which made out of Sanskritic language. considering the fact that 1950 Sanskritic language has been composed with the Devanagari letter set.

To the most trustworthy of our facts widespread dataset for Sanskritic language numeral is not accessible until in recent times. alongside these traces, dataset of Sanskritic language composed numerals 0 to 9 is formed by using accumulating the composed documents from scholars. information grouping is accomplished on a sheet uncommonly meant for gaining knowledge of combination. Essayists from entirely sudden callings had been picked collectively with understudies, representatives, teachers, and sellers and had been approached to scribble down the numerals. No imperatives were obligatory on the use of ink or pen aside from that they have to scribble down the numerals inside the cases of the sheets gave to them.

A well-characterized include extraction algorithmic popular makes the grouping technique gradually right down to earth and low-cost. 2 well-characterised methods for highlight extraction utilized in our strategy are Fourier descriptors and standardized chain codes. spotlight extraction arrange in man or woman acknowledgment, as in any example acknowledgment mission, assumes a big job in elevating the notoriety exactness. The alternatives are extricated from parallel characters. in this manner, the qualities applied for association lie on my own in the structure sorts. The few characters are comparative in shape or slight range in the magnificence should end result into 
misclassification. The options hand-picked should deal with of these problems. further, one element extraction and arrangement level may want to recognize a man or woman which can't be perceived by the contrary detail extractor and classifier blend. in this way a half and $1 / 2$ framework is required a good way to understand the characters over a massive fluctuate of variable conditions. The edited characters in every auxiliary magnificence are resized to a company size before extricating the alternatives. three very unexpected options are removed to be related to multiple remoted neural structures. The selections extricated are photo issue thickness picks, Euclidian separation selections and changed estimate wave choices. The options extraction strategy is clarified straightaway.

\section{LITURATURE SURVEY}

Acknowledgment of Marathi Manually written Numerals by way of utilizing Bolster Vector system in this paper with the aid of Ms. Pratibha V. Waje we generally tend to blessing a approach for programmed acknowledgment of Marathi composed numerals. The factor of composed numeral acknowledgment (HNR) framework is to institution enter numeral collectively of $\mathrm{k}$ training. Transcribed numeral acknowledgment framework have 2 section spotlight investigation and example order The element extraction is by way of abuse the chain code and Fourier Descriptor we have a tendency to test the type of numerals . in the wake of preprocessing the numeral photo, the standardized chain code and furthermore the Fourier descriptors of the shape of the numeral territory unit extricated. those alternatives territory unit at that point sustained within the assist Vector device (SVM) for arrangement. The proposed machine is probed statistics of 12690 examples of Marathi composed numeral abuse quintuple move approval method. we have gotten acknowledgment exactness of $95.12 \%$.

The according that a cost-effective method for recognition of remoted Marathi numerals has been conferred with strain on feature extraction technique. Density supported division approach, and critical moments place unit used as alternatives to facilitate popularity method. Experiments vicinity unit dispensed on a info length of 12690 numeral photographs victimization multiple cross validation technique. The projected device is freelance of variability involved within the literary style of numerous people and is dilution free. popularity rate received with guide Vector system (SVM) classifier $(97.89 \%)$ is satisfactory as compared with minimum distance classifier (ninety six.31 \%) and ok-Nearest Neighbor (okay-NN) classifier (ninety six.fifty five\%) on this paper we have a tendency to blessing a format of spotlight Extraction strategies for disconnected acknowledgment of constrained Gurumukhi characters acknowledgment. decision of highlight Extraction machine is maybe the one most large take into account conducting superior in instance acknowledgment. Our paper affords region based totally for the maximum component approach with the intention to be that the blend of image focal point of mass quarter and area focal point of mass region of numeral/character photograph. In photograph focus of mass zone character is a component into $n$ equal region so image focal point of mass and as a result the regular proper approaches from man or woman focal factor of mass to every area/matrix/encloses blessing photograph is determined. Correspondingly, in area attention of mass quarter character photograph is a part into $n$ equivalent zones and focal point of mass of each quarter/bins/matrix and everyday exact ways from region focal point of mass to every constituent blessing in square/zone/lattice is decided. we've applied SVM and $\mathrm{k}-\mathrm{NN}$ for later classifier and acknowledgment reason. We tend to were given ninety $5.11 \%$ and ninety.sixty four\% acknowledgment precision SVM and k-NN severally. Malika Ait Aider et.al. Kamal Hammouche et.al., Djamel Gaceb et.al. (2018) rumored that approach of written person reputation which combines a moving ridge remodel and one assist vector system classifier. The moving ridge remodel allows u.s.a. characterizing the person pictures through a set of alternatives. The connexion of those alternatives relies upon powerfully on the selection of the sort of the shifting ridge and sub-pics derived from the moving ridge transform. in the course of this paper, many exams collectively with many transferring ridges and swish and information sub-images derived from the wavelets square measure carried out so as to see the handiest wavelet and therefore the nice sub-photograph within the written reputation framework. Experimental consequences on MNIST information screen that sym8 moving ridge outperforms alternative sorts of wavelets as those hired within the previous works. They show conjointly that options extracted from the sleek sub-photo allowed reaching the best popularity fee. The projected approach is reasonably priced compared with opportunity written reputation strategies revealed within the literature. As future paintings, we have a tendency to mean on one hand to combine a standardization operation as pre-processing technique with the intention to manipulate the placement and shape of man or woman photos, hence on cut back form variation between the photos of identical class. On alternative hand, to analyze opportunity alternatives extracted from the four sub-pics.

Teena Mittal and Rajendra Kumar Sharma (2015) reported that speaker-dependent, remoted phrase recognition gadget for Hindi numerals has been enforced. options of speech in terms of LPC, MFCC and combination of LPC and MFCC place unit concept-approximately to renowned Hindi numerals. The SVM classification is achieved in 2 steps. initially, a one-versus-all SVM classifier is hired to identify the Hindi language then ten one-versus-all classifiers location unit accustomed well known Hindi numerals. The experiments are carried out in 2 levels. In preliminary component, the amount of frames has been fastened and definitely distinctive folds in $\mathrm{k}$-fold move validation are implemented for coaching and trying out of SVM. To discover the best kernel method, linear, polynomial and RBF kernels are used for the improvement of SVM. The very satisfactory reputation fee of ninety $4.0 \%$ has been achieved exploitation linear kernel strategy with combination of LPC and MFCC alternatives and 10-fold move validation. The linear kernel approach systematically ruled opportunity kernel strategies throughout this part of test. The linear kernel has gain compared to opportunity kernels is that it would not

Published By: 
want any kernel parameter to line. inside the second a part of experimentation, the amount of frames has been numerous to calculate the popularity charge exploitation SVM with linear kernel. The very pleasant recognition price of $96.8 \%$ may be carried out with combination of LPC and MFCC alternatives, whereas the use of 10 -fold pass validation and twenty six frames.

\section{1 want of labor:}

To obtain higher consequences in forensic packages: The current utility area of the framework is used in crime research and prosecution. We anticipate a huge potential for studies and development inside the field of forensic handwriting and signature analysis as well as creator identification. Humanoid: This application is used to do away with the human mistakes in handwriting. If we are using antique papers which aren't seen well, we will use this approach.

Robotics: Humanoid robots were used in several scientific regions as a research and development tools. similarly to research, humanoid robots were developed to perform exceptional human tasks like a human. they are reprogrammable time and again for pleasurable distinct responsibilities anticipated from them. With the advancements on technology, humanoid robots had been widely used on industrial applications because of their lowering prices. on this take a look at, synthetic neural community application were implemented on photographs which taken from NAO's digital camera instantly. used to discover characters. NAO robotic has been successful to discover and say characters on images after training robot with schooling dataset. The consequences show that, using neural community algorithms offers true outcomes on man or woman reputation programs.

\section{METHODOLOGY \& RESULTS}

For any individual recognition machine, there are four foremost ranges namely pre-processing, Segmentation, function extraction, category and reputation.

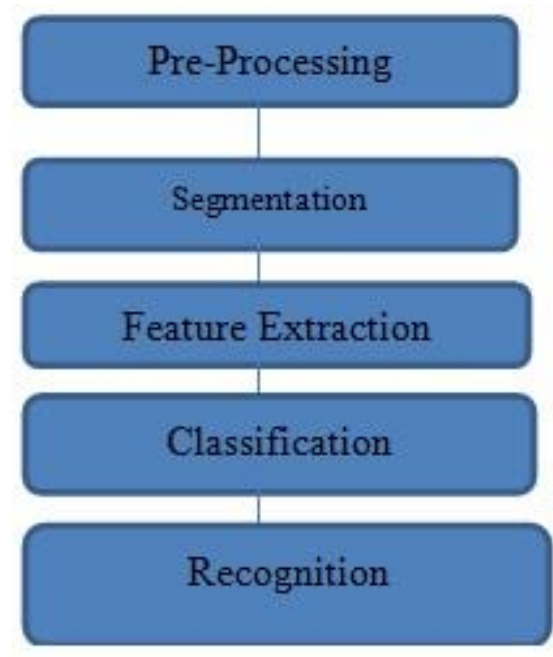

Fig.1 Character Recognition System

stage 1: person Acknowledgment Framework: we use Insect Excavator Calculation.

degree 2: highlight Extraction: To get rid of the factor vectors from the standardized images, a sliding window Segmentation and feature extraction techniques have been

method is utilized. The seven highlights are the accompanying:

1. The mean dim estimation of pixels

2. the point of interest of gravity of the pixels

three. the second one-request vertical snapshot of the focus of gravity

4. The places of the best and lowermost square of the pixels

five. The pace of development of these positions

6. the quantity of darkish white advances between the highest and lowermost pixels

7. The quantity of darkish pixels among the highest and lowermost pixels

stage three: Intermittent Neural device:

An Recurrent Neural network:(RNN) is associations of version containing a self-related shrouded layer. RNNs give a rich approach for managing consecutive facts that typifies connections be tween's statistics focuses which are near inside the succession .RNN can utilize past placing.

\section{CONCLUSION}

The success of deep studying based in the main fashions have focused on recent architectures and consequently the benefit of vast scale annotated know-how. at some point of this work, we will be inclined to discover those 2 factors systematically for up written popularity for scanned off-line report images. We have a tendency to suggest a changed CNN-RNN hybrid design with a big specialize in effective training using: (i) economical layout of community mistreatment artificial information for pertaining, (ii) image standardisation for slant correction and (iii) domain unique expertise transformation and distortion for studying crucial invariances. We will be inclined to perform a close ablation examine to examine the contribution of person modules and present nation of art effects for the task of at liberty line and phrase reputation on well-favored datasets like IAM, RIMES and GW.

\section{REFERENCES}

1. Shailendra Kumar Shrivastava,Sanjay Gharde," help Vector machine for Handwritten Devnagari Numeral recognition",worldwide magazine of laptop programs, extent 7, No.eleven, October 2010.

2. S.M.Mali," more than one feature Extraction techniques for Handwritten Marathi Numeral popularity",worldwide magazine of Engineering studies and era,volume 2, difficulty 10,October 2013.ISSN: 2278-0181.

3. three. Gurpreet Singh,Rajdeep Kaur,'online Handwriting reputation machine for English Language the use of assist Vector gadget technique",international journal of strengthen studies in laptop technology,extent 9,No.2,March-April 2018.

4. S.M.Mali,"second and Density primarily based Handwritten Marathi Numeral popularity",Indian magazine of computer technology and Engineering,Vol.3,No.five,Oct-Nov.2012.

5. Vinita H. Patil," recognition of Numerals using SVM and Blob Classifier", international magazine of revolutionary studies in pc and communique Engineering, Vol. four, 
problem 7, July 2016.

6. Sandhya Arora, Debotosh Bhattacharjee, Mita Nasipuri, L. Malik, M. Kundu and D. k. Basu," overall performance evaluation of SVM and ANN for Handwritten Devnagari man or woman reputation", global magazine of pc technology issues, Vol. 7, trouble 3, can also 2010.

7. Malika Ait Aider, Kamal Hammouche, and Djamel Gaceb," popularity of Handwritten Characters based on Wavelet remodel and SVM Classifier", The worldwide Arab magazine of facts era, Vol. 15, No. 6, November 2018.

8. A A Jinturkar, Dr. P.B. Khanale," issues and generation Evolution in Handwritten sNumeral recognition of Marathi", magazine of Engineering computers \& carried out Sciences (JECAS) volume 6, No.12, December 2017. ISSN No: 2319-5606

9. nine. Gajanan Birajdar and Mansi Subhedar," USE OF JPEG algorithm IN HANDWRITTEN DEVNAGRI NUMERAL popularity", worldwide magazine of dispensed and Parallel systems (IJDPS) Vol.2, No.4, July 2011.

10. Gita Sinha, Mrs. Rajneesh Rani, Prof. Renu Dhir," Handwritten Gurmukhi person popularity the usage of ok-NN and SVM Classifier", global magazine of superior volume 2, trouble 6, June 2012. ISSN: 2277 128X

11. Teena Mittal1 and Rajendra Kumar Sharma2," Multiclass SVM primarily based Spoken Hindi Numerals reputation", The worldwide Arab journal of statistics era,

12. PANKAJ KALE, 2ARTI V. BANG, 3DEVASHREE JOSHI," recognition OF HANDWRITTEN DEVANAGARI CHARACTERS using gadget learning approach", worldwide magazine of business Electronics and electrical Engineering, volume-three, issue-nine, Sept.-2015. ISSN: 2347-6982

13. M. J. Bahetil and okay. V. Kale2," contrast OF SVM AND FUZZY CLASSIFIER FOR AN INDIAN SCRIPT", ICTACT journal ON tender COMPUTING, JANUARY 2012, quantity: 02, issue: 02. ISSN: 2229-6956(online)

14. Arjun Singh1, Kansham Angphun Maring2," Handwritten Devanagari man or woman recognition using SVM and ANN", international journal of advanced studies in computer and verbal exchange Engineering, Vol. four, issue 8, August 2015. ISSN (online) 2278-1021.

15. Ms. Pratibha.V. Waje1, Ms.Mrunalinee N. Shirole2, Prof.Sandeep Sahu3 ,Dr.R.S.Patil4," automated reputation of isolated Marathi Handwritten Numerals", IOSR magazine of Engineering, Apr. 2012, Vol. 2(4) pp: 697-703.

16. Mustafa S. Kadhm, Dr. Alia Karim Abdul Hassan," Handwriting word reputation primarily based on SVM Classifier", worldwide journal of superior laptop technological know-how and packages, Vol. 6, No. eleven, 2015.

17. Gauri Katiyar1, *, Ankita Katiyar2, Shabana Mehfuz3," Off-Line Handwritten character popularity device using assist Vector machine", American journal of Neural Networks and packages, ISSN: 2469-7400 (Print); ISSN: 2469-7419 (on-line), 2012.

18. Jos'e Carlos Aradillas," Boosting Handwriting text knowledge of",Apr.2018

19. Arindam Chowdhury, Lovekesh Vig," An Efficient stop-to-end Neural version for Handwritten text popularity",2018.

20. Salma Shofia Rosyda1 and Tito Waluyo Purboyo2," A evaluation of diverse Handwriting reputation techniques", international journal of carried out Engineering research ISSN 0973-4562 volume 13, wide variety 2 (2018) pp. research in laptop science and software Engineering, Vol. 12, No. 6A, 2015. popularity in Small Databases with switch gaining

1155-1164.

21. Khaled S. Younis1," ARABIC HANDWRITTEN person reputation based ON DEEP CONVOLUTIONAL NEURAL NETWORKS", Jordanian magazine of computers and statistics generation (JJCIT), Vol. 3, No. three, December 2017.

22. Karishma Verma, Dr. Manjeet Singh," A Literature Survey on Handwritten man or woman popularity the use of Convolutional Neural community", global journal of management, generation And Engineering,2014 ISSN NO :2249-7455.

23. Yi-Chao Wu1,2, Fei Yin1, Zhuo Chen1,2, Cheng-Lin Liu1,2," Handwritten chinese language textual content popularity the usage of Separable Multi-Dimensional Recurrent Neural community", 2017 14th IAPR international convention on file analysis and reputation.

24. Emilio Granell 1,* id , Edgard Chammas 2, Laurence Likforman-Sulem 3, Carlos-D. Martínez-Hinarejos 1, Chafic Mokbel 2 and Bogdan-Ionu,t Cîrstea 3," Transcription of Spanish ancient Handwritten documents with Deep Neural Networks", acquired: 30 October 2017; generic: 2 January 2018; published: 11 January 2018.

25. Zheheng Rao1,2, Chunyan Zeng1,2, Minghu Wu1,2*, Zhifeng Wang3, Nan Zhao1,2, Min Liu1,2 Xiangkui Wan1," studies on a handwritten character popularity algorithm based on an extended nonlinear kernel residual community", KSII TRANSACTIONS ON internet AND information systems VOL. 12, NO. 1, January 2018.

26. S M Shamim, Mohammad Badrul Alam Miah, Angona Sarker, Masud Rana \& Abdullah Al Jobair," Handwritten Digit recognition the use of device gaining knowledge of Algorithms", worldwide journal of pc technology and technology: D Neural \& synthetic Intelligence extent 18 issue 1 model 1.0 yr 2018

27. PrashanthVijayaraghavan,"HandwrittenTamilRecognitio nusingaConvolutionalNeuralNetwork",2012.

28. Karishma Verma1*, Manjeet Singh2," Hindi Handwritten person recognition the use of Convolutional Neural network", worldwide magazine of computer Sciences and Engineering, Vol.-6, issue-6, June 2018. E-ISSN: 2347-2693.

29. V. S. Dhaka, Manoj Kumar, Harshit Sharma," person reputation of Offline Handwritten English Scripts: A evaluate", special conference difficulty: country wide convention on Cloud Computing \& large facts, 2010.

30. Framework for privateness preserving category in records Mining ,DYK Sharma, GM Sharif, journal of emerging technology and progressive research 5 (nine), 178-183

31. overall performance evaluation of put off Tolerant Networks Routing Protocols underneath varying Time of live, VK Samyal, DYK Sharma international magazine of enhance studies in laptop technology (IJARCS) 8

32. $\mathrm{Li}-\mathrm{Fi}$ the maximum latest Innovation in wireless communication S Saini, DYK Sharma global journal of advanced studies in pc science and software.

33. net web page type on news Feeds using Hybrid approach for Extraction ad Patel, DYK Sharma facts \& communique technology for shrewd device 107 (6), 399-405.

34. effect of buffer size on exceptional drop guidelines (DLR, MOFO and E-Drop) for MaxProp Routing Protocol in DTN VK Samyal, DYK Sharma worldwide magazine of studies in implemented technology \& Engineering

35. a pragmatic assessment of pressure and overall performance trying out technology for net based totally packages P sonkari, DYK Sharma IEEE, Amity 
international conference on artificial Intelligence (AICAI), 399-403

36. impact of network Load and node Mobility at the overall performance of Proactive, Reactive and Hybrid routing protocols of MANET V Singla, YK Sharma worldwide journal of advanced research in laptop technological know-how 8 (3

37. evaluation of egocentric Node conduct in delay Tolerant Networks Routing Protocols VK Samyal, DYK Sharma international magazine of revolutionary research in science and Engineering

38. importance observe OF person internet access information MINING FOR commercial enterprise INTELLIGENCE, advert Vyas, YK Sharma Indian magazine of implemented research

39. A complete study ON type OF automated CATEGORIZATION OF net sites: A PROPOSED technique DA Vyas, DYK Sharma INDIAN magazine OF applied studies (IJAR) forty. using Open CV for gadget studying in actual Time laptop vision and photo ProcessingP S, YK SharmaInternational magazine of new technology and Engineering 